\title{
ZS Research Square \\ Depression and quality of life in patients with inflammatory bowel diseases
}

Jacqueline Sundström ( $\nabla$ jacqueline.sundstrom@regionstockholm.se )

Center for Clinical Research, Sörmland

Sol-Britt Rahm

Center for Clinical Research, Sörmland

\section{Research Article}

Keywords: Depression, inflammation, IBD, quality of life

Posted Date: March 2nd, 2022

DOI: https://doi.org/10.21203/rs.3.rs-1327361/v1

License: (9) This work is licensed under a Creative Commons Attribution 4.0 International License. Read Full License 


\section{Abstract}

This cross-sectional study was carried out to find prevalence and possible relation between decreased quality of life and depression among patients with inflammatory bowel disease.

Aims: Due to possible common biological pathways between inflammation in IBD and depression and due to the high burden of depression on both patient and health care institution, it is important to identify and initiate treatment to improve quality of life, disease outcome and reversed events associated with depression.

Methods: 41 patients with either Crohn's disease or ulcerative colitis participated in a questionnaire-based interview. Beck's depression inventory was used for depression assessment and quality of life. Peripheral blood was collected for analyzing inflammatory and anemia markers. Data was then used for statistical analysis.

Results: most patients were clinically free of depression. However, there was a strong correlation between number of exacerbations and depression in patients with Ulcerative Colitis, there was a negative correlation between serum leukocytes and depression, and the use of Mesalazine had a protective effect.

Conclusion: inflammation in the gut may provoke depressive phenotype and vice versa. This may be caused by biological, social and psychological factors. Depression may also cause decreased quality of life, why it is necessary to identify these symptoms early and thereby reduce the risk of decreased quality of life.

\section{Introduction}

Inflammatory bowel disease (IBD) is a group of chronic, multisystem, immune-mediated diseases of the gastrointestinal tract (GIT), mainly comprising Crohn's disease (CD) and ulcerative colitis (UC). The diseases are typically manifesting in a remitting and relapsing course.

The intestinal manifestation of the disease differs, CD causes a transmural inflammation with oedema and ulceration in any part of the GIT (from mouth to anus). UC result in a superficial inflammation of the mucosa and submucosa of the colon with subsequent erosion and ulceration, starting distally and in an ascending manner. CD may manifest with a non-bloody chronic diarrhoea and abdominal pain, whereas UC patients typically present with crampy abdominal pain with bloody diarrhoea containing mucoid material.

The mucosal barrier seems to be disrupted in patients with IBD, which leads to increased permeability to microbial particles, and they in turn when displaced, may cause an inflammation [1].

The resultant inflammation will be uninhibited and continued due to pro-inflammatory mediators release such as tumour necrosis factor (TNF)- $a$ and interleukins 6 and 10 (IL-6 and IL10). The inflammation response can be detected by blood samples, including IL, SR, CRP and LPK as well as anemia. 
Depression could provoke IBD development [2]. Depression is a complex disorder, proposed to involve genetic, epigenetic, psychological, and environmental factors that decreases a person's capacity to tolerate stress [3]. The symptoms characteristic for the syndrome is persistent feeling of sadness, worthlessness, and hopelessness. Patients typically show changes in cognitive function (commonly concentration disturbances), appetite, sleep-pattern, they tend to become more easily irritable, and lose interest in previously pleasurable activities, also known as anhedonia. This may further lead to social withdrawal, suicidal thoughts and in worst case, suicide [3].

Several mechanisms have been implicated in the pathophysiology of depression, including alterations in brain neurotransmission, decreased neuroplasticity and neurogenesis in the limbic system (among others), as well as vascular and inflammatory changes in the CNS. All mentioned changes are not seen in every patient, but it is believed that the different pathophysiological mechanisms are interconnected, and alteration in any part may initiate a series of events and biological effects causing depressive symptomatology [4].

Up to $80 \%$ of patients with IBD and comorbid depression were diagnosed with depression more than two years before they were diagnosed with IBD. Of them, a higher proportion had an earlier onset of IBD than those without depression. This suggest that the illness itself may not only be a trigger of depression, but also that an underlying depression may be a trigger to develop IBD [2].

Studies have shown that patients with IBD that had active disease [5], increased amounts of exacerbations [6], higher disability related to bowel disease [7] and who were using corticosteroids $[3,7,8]$ had higher rates of comorbid depression, and patients with inactive disease had approximately the same rate as in healthy controls [2]. This further leads towards the thought that biological mechanisms in the active inflammation play a big role in the development of depression in these patients.

Studies of the U.S. population have shown that the prevalence of depression in IBD subjects was as high as $49 \%$ compared to $23 \%$ in non-IBD subjects. The rates increased with female gender, older age and if the patient was separated, divorced or widowed [9]. In addition, it was also possible to see a connection with higher rates of depression in patients who were less physically active and those who had lower educational level [8], or lack of family support [10].

The relationship between depression and IBD seem to be bidirectional, as suggested by the two-fold increased risk of developing CD in patients with depression, compared to that of non-depressed individuals.

An active inflammation in the gut leads to stress response, which is an important contributor to the development of depression. Both emotional and physical stress triggers the noradrenergic system (NE) which in turn activate the hypothalamic-pituitary-adrenal (HPA) axis. [4]. Other causes of a stress response include early childhood negative events (such as reject or neglect), as well as 'loss of autonomy', a state that may occur when a person falls ill in a medical illness [11]. Major depressive 
disorder is associated with increased CRP and TNF-a. Antidepressants such as selective serotonin reuptake inhibitors (SSRIs) normalize levels of CRP and IL-6 according to a meta-analysis [12].

Quality of life (QoL) is decreased in patients with IBD, which leads to poorer compliance to treatment and thus worse outcome of disease [9]. It has been shown in the Canadian population that decreased QoL, depression and anxiety in patients with IBD were associated with younger age, female sex, and those who were single [9].

In addition, the chronic course of the disease requires life-long contact with the health care system, tight adherence to medical treatment and an ability of self-management. The perceived disability and psychological stress may therefore trigger negative mental well-being and reduced ability in coping with the disease [5]. Over time, the ability of self-management may be reduced leading to more symptoms, more pain, more stress, and less ability to engage in activities, which may eventually further intensify negative emotions. In this aspect, good control of symptoms is exceedingly important for the physical as well as the mental well-being $[5,7]$.

Previous studies have concluded that having a medical illness increased the risk of developing depression by $5-10$ times compared to that of healthy subjects [13].

Due to this knowledge, it can be concluded that depression and decreased mental well-being in patients with IBD should be identified, in order to minimize further harm to the patient, to inprove the disease outcome and quality of life, and to decrease the burden of the healthcare system.

\section{Methods}

A cross-sectional study was conducted on forty-one patients with either Crohn's disease or Ulcerative colitis, recruited from Riga East Clinical University Hospital (RECUH) stationary "Gailezers", Gastroenterology, Hepatology and Nutrition Clinic in Latvia.

The study was conducted in 15 months and the patients in the clinic were asked to participate in outpatient interviews. Patients who met all inclusion criteria were included in the study.

\section{Inclusion criteria:}

1. Patients who agreed to participate and had signed written informed consent,

2. Out-patients,

3. Ages between 18-80,

4. CD patients with clinically, endoscopically, and histologically confirmed diagnosis,

5. UC patients with clinically, endoscopically, and histologically confirmed diagnosis. 
The questionnaire included information regarding the patient and disease, including treatments used, complications and hospitalizations. Thereafter peripheral blood was collected to detect anaemia $(\mathrm{Hb})$ and indicators for inflammation (CRP, Leukocytes, IL-6, and IL-10). Subsequently, severity of disease could be evaluated using the Crohn's disease activity index (CDAl) and for Ulcerative colitis (Mayo score).

Beck's depression Inventory (BDI) was used for assessment of depressive symptoms and QoL.

\section{Statistical analysis}

A statistical analysis was performed using IBM SPSS version 22.0. Descriptive statistics were displayed as frequencies, proportions, mean values and standard deviations and used to analyse clinical and demographic data. For analytical statistics, nonparametric tests were used; spearman's correlation analysis and Mann-Whitney test for the comparison of groups. P-value $<0.05$ was considered statistically significant.

\section{Results}

Seven patients had CD, 4 of them were males and 3 of them were females. 34 patients had UC whereas 21 were males and 13 females. Among patients with CD, 6 of them had been treated with conservative treatment including mesalazine and corticosteroids and 3 of them had been at least once been treated surgically. Among patients with UC, 21 were 21 treated with conservative treatment, 7 of them with corticosteroids, 11 with sulfasalazine, 17 with mesalazine, 5 with azathioprine and 2 patients with biological medications. Of them 2 patients were treated surgically.

In patients with $C D, 11$ of them had had exacerbations. Four patients suffered from intestinal complications and two from extra-intestinal complications.

Of patients with UC, three patients had had exacerbations and three had suffered from intestinal complications and one from extra-intestinal complications. Of patients with UC, 22 had clinically mild disease, 8 had moderate disease activity and four were in clinical remission. None of patients had severe disease activity.

Of CD patients, three were previously hospitalized and of UC patients had 10 been previously hospitalized. Of patients with $C D$, all were in clinical remission.

\section{BDI scores (depression) and associations}

The median BDI score in all patients, mean value is $7.6(S D=9)$. There was no statistically significant difference in BDI score between patients with CD compared to patients with UC. Most of the patients were clinically free from depression, and only one patient had moderate depression according to BDI (Fig. 1). 
There was a strong correlation between numbers of exacerbations and higher IBD scores in patients with $\mathrm{UC}(\mathrm{p}=<0,001 ; r=0,135)$.

There was a negative correlation between serum leukocytes and BDI scores $(p=0,032)$. The use of Mesalazine had protective effect $(p=0,032)$

Age, gender, previous hospitalizations, the use of corticosteroids, sulfasalazine, biologic treatment, previous surgical therapy, serum CRP, IL-6, IL-10 and hemoglobin concentration had no correlation with distribution of IBD scores. Neither had intestinal complications.

\section{Quality of life (QoL) score and associations}

Mean QoL score among IBD patients was 49,95 (SD 14,07), maximum score 70 and minimum score 0 . In CD patients the mean score was 46,26 (SD 15,12) and in UC patients the mean score was 50,7 (SD13,97). There was no significant difference in QoL score between CD and UC patients.

There was a significant negative correlation between BDI and QoL $(p<0,001 ; r=-0,536)$, the higher the BDI score the lower the QoL (Fig. 2), and the mean QoL score was significantly lower in females $(14,91)$ than in males $(24,90)(p=0,009)$.

A moderate correlation could be seen between serum II- 6 and QoL score $(p=0,034 ; r=-0,409)$. Neither age, disease severity, number of exacerbations, number of previous hospitalizations, or whether the patients were using surgical or conservative treatment had any relevancy with QoL.

\section{Discussion}

Our hypothesis is that patients with IBD have affected QoL and risk of developing depression, perhaps due to biological interplay between inflammation in the gut and inflammatory, hormonal and structural changes in the brain.

There was a strong correlation between the number of exacerbations and decreased mood in UC patients which leads to the thought that both biological and psychological factors are likely to be the cause of comorbid depressive symptomatology. It also can give clues, that the low number of participants gives faulty result of activity and severity of disease as independent contributing variables to depressive symptomatology.

In this cross-sectional study, most patients had no depressive symptoms, and only one patient had moderate depressive symptoms. This may or may not be explained by the fact that most patients had mild disease, and in $\mathrm{CD}$, all were in clinical remission. In addition, only a low number of patients had complications related to disease.

When we evaluated the lab values, we found a negative correlation between leukocyte count and depressive symptomatology. This might substantiate the theory of inflammation causing long term structural changes in the brain, and therefore no active inflammation is necessary for the depression to 
be expressed, and also might suggest the increased time needed for the brain tissue to recover from effects of repeated inflammatory insult to the gut.

Quality of life has been reported to be reduced in patients with IBD in other studies. In this study, median quality of life in patients with $C D$ was higher. Several factors may explain such result, for example all patients were in clinical remission. An increasing BDI showed to have lower QoL scores, which may also implicate the psychological stress in coping with the disease, the perceived disability and the need for continuous contact with the health care system.

Due to the possible dramatic effects of persistent and recurrent inflammation in the gud and hence the potential complications in the brain, it is exceedingly important to view the patient in a holistic perspective, to identify decreased mental well-being, and to treat it. Depression is not only a harm to the patient but is also a huge burden to the healthcare system, and communication between different specialities could result in a better outcome for both patients, and the health-care system. Use of a QoLmonitor in patients with IBD patients might result in a more frequent discussion of QoL-topics, associated with high levels of patients' satisfaction.

\section{Declarations}

\section{Ethics approval and consent to participate:}

Each patient included in the study has given informed consent to participate in a questionnaire-based interview as well as given blood for analysis of parameters included in the "materials and methods" section. All methods were carried out in accordance with regulations. The collection of data and the use for statistical analysis is approved by the ethics committee of Riga Stradins University on the 10.9.2015, led by professor Olafs Bruvers.

\section{Consent for publication:}

not applicable

\section{Availability of data and materials:}

The datasets generated and analysed during the current study are not publicly available for the protection of personal data, but are available from the corresponding author on reasonable request.

\section{Competing interest:}

none 


\section{Funding:}

Not applicable

\section{Authors' contributions:}

Solbritt and Jacqueline wrote the main manuscript text. Jacqueline prepared figures 1 and 2 . All authors reviewed the manuscript.

\section{Acknowledgements:}

Many thanks to dr. Vita Skuja for your inspiration, support and invaluable assistance in this project.

\section{References}

1. Ahluwalia, B., Moraes, L., Magnusson, M. K., Ohman, L. Immunopathogenesis of inflammatory bowel disease and mechanisms of biological therapies. Scandinavian Journal of Gastroenterology, 2018, DOI: 10.1080/00365521.2018.1447597

2. Bernstein, C. N. Psychological stress and Depression: Risk factors for IBD? Digestive diseases, 2016, Vol. 34, No. 1-2, p. 58-63.

3. Mooney, C. 2013. Depressive disorders: diseases and disorders. San Diego: ReferencePoint Press, Inc, p. 8-73.

4. Dean, J., Keshavan, M. The neurobiology of depression: An integrated view. Asian Journal of Psychiatry, 2017, Vol. 27, p. 101-111.

5. Keefer, L., Kane, S. V. Considering the Bidirectional Pathways Between depression and IBD: Recommendations for Comprehensive IBD Care. Gastroenterology and Hepatology, 2017, No 3, Vol. 13, p. 164-169.

6. Nowakowski, J., Chrobak, A. A., Dudek, D. Psychistric illnesses in inflammatory bowel diseases psychiatric comorbidity and biological underpinnings. Psychistria Polska, 2016, Vol. 50, Nr 6, p. 1157-1166.

7. Chan, W., Shim, H. H., Lim, M. S., Sawadjaan, F. L. B., Isaac, S. P., Chuah, S. W., Leong, R., Kong, C. Symptoms of anxiety and depression are independently associated with inflammatory bowel disease-related disability. Digestive and Liver diseases, 2017, vol. 49, p. 1314-1319.

8. Long, M. D., Kappelman, M. D., Martin, C. F., Chen, W., Anton, K., Sandler, R. S. Risk factors for depression in the elderly inflammatory bowel disease population. Journal of Crohn's and Colitis, 2014, Vol. 8, p. 113-119.

9. Byrne, G., Rosenfeld, G., Leung, Y., Qian, H., Raudzus, J., Nunez, C., Bressler, B. Prevalence of Anxiety and Depression in Patients with Inflammatory Bowel Disease. Canadian Journal of Gastroenterology 
and Hepatology, 2017, CONTINUE TO FIND??? Don't see it

10. Bhandari, S., Larson, M. E., Kumar, N., Stein, D. Association of Inflammatory Bowel Disease (IBD) with Depressive Symptoms in the United States Population and Independent Predictors of Depressive Symptoms in an IBD Population: A NHANES Study. Gut and Liver, 2017, No 4, Vol. 11, p. 512-519.

11. Stringer, S., Church, L., Davison, S., Lipsedge, M. 2009. Psychiatry: Principles, Reality, Next Steps. First edition. New York: Oxford University Press Inc, p. 31-56.

12. Krishnadas, R., Cavanagh, J. Depression: An Inflammatory illness? Journal of Neurology Neurosurgery and Psychiatry, 2012, Vol. 83, p. 495-502.

\section{Table}

Table 1

Interpretation of BDI scores. Maximum score is 63

BDI scores

\begin{tabular}{ll}
\hline $0-10$ & Ups and Downs are considered normal \\
\hline $11-16$ & Mild mood disturbance \\
\hline $17-20$ & Borderline clinical depression \\
\hline $21-30$ & Moderate depression \\
\hline $31-40$ & Severe depression \\
\hline$>40$ & Extreme depression
\end{tabular}

\section{Figures}




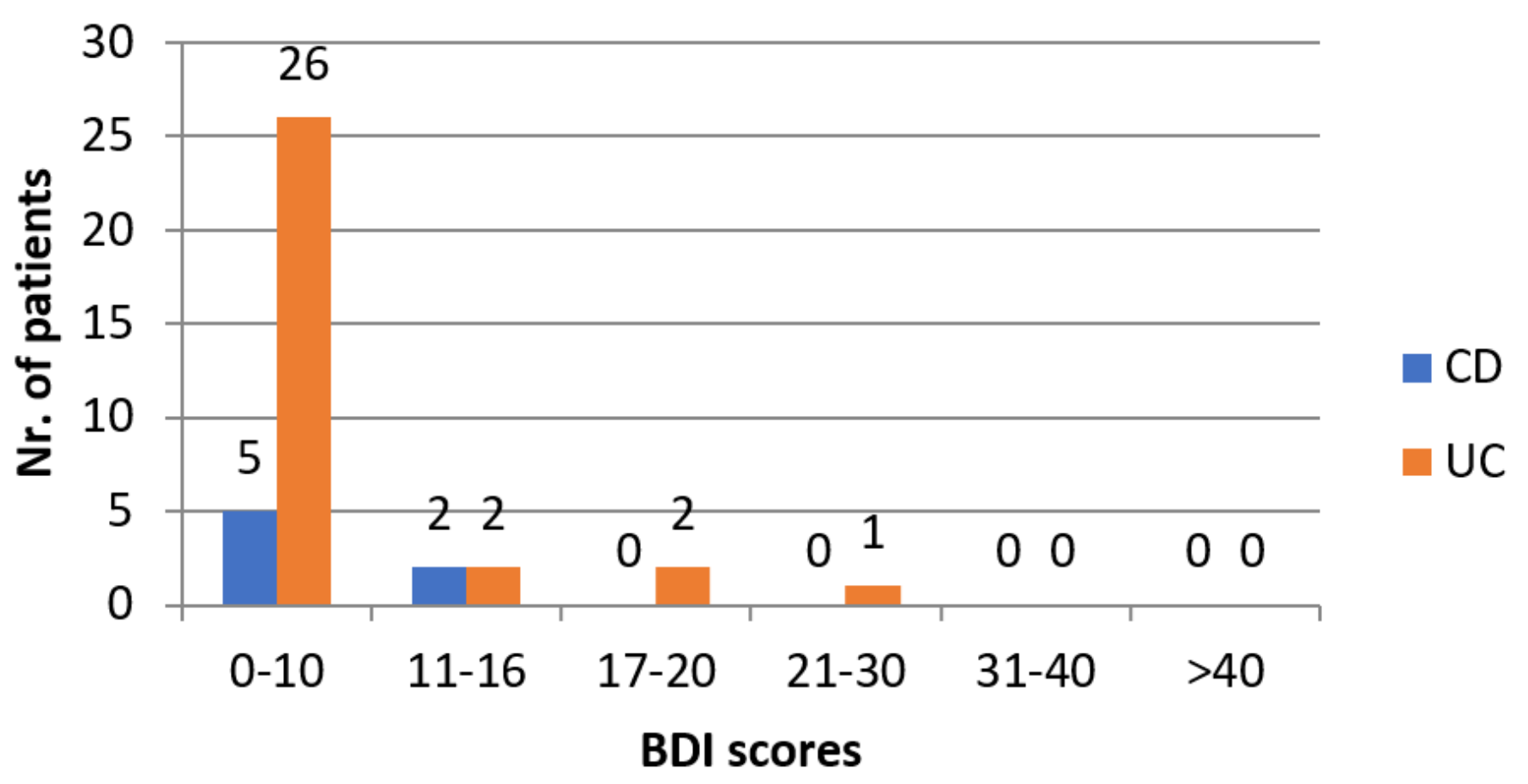

Figure 1

Distribution of BDI scores in all patients devided between $C D$ and UC 


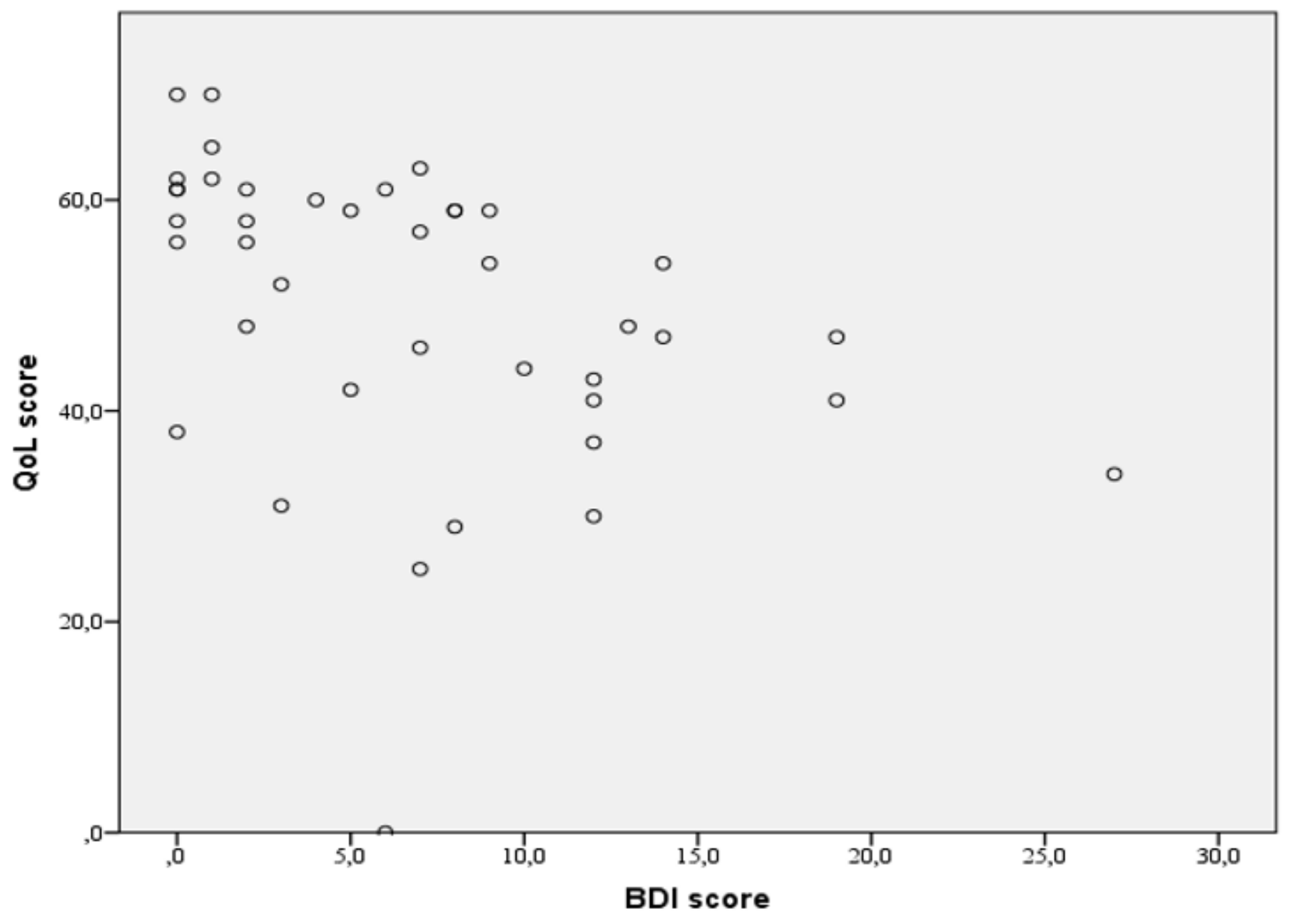

Figure 2

Graph showing negative correlation between BDI score and QoL 